\title{
Odour coding is bilaterally symmetrical in the antennal lobes of honeybees (Apis mellifera)
}

\author{
C. Giovanni Galizia, Karl Nägler, Bert Hölldobler ${ }^{1}$ and Randolf Menzel \\ Institut für Neurobiologie, Fachbereich Biologie, Freie Universität Berlin, D-14195 Berlin, Germany \\ ${ }^{1}$ Theodor Boveri Institut der Universität, Lehrstuhl für Verhaltensphysiologie und Soziobiologie der Universität (Zoologie II), \\ Am Hubland, D-97074 Würzburg, Germany
}

Keywords: development, insect, olfactory glomeruli, optical recording, statistical analysis

\begin{abstract}
The primary olfactory neuropil, the antennal lobe $(A L)$ in insects, is organized in glomeruli. Glomerular activity patterns are believed to represent the across-fibre pattern of the olfactory code. These patterns depend on an organized innervation from the afferent receptor cells, and interconnections of local interneurons. It is unclear how the complex organization of the $A L$ is achieved ontogenetically. In this study, we measured the functional activity patterns elicited by stimulation with odours in the right and the left AL of the same honeybee (Apis mellifera) using optical imaging of the calcium-sensitive dye calcium green. We show here that these patterns are bilaterally symmetrical ( $n=25$ bees). This symmetry holds true for all odours tested, irrespective of their role as pheromones or as environmental odours, or whether they were pure substances or complex blends $(n=13$ odours). Therefore, we exclude that activity dependent mechanisms local to one AL determine the functional glomerular activity. This identity is genetically predetermined. Alternatively, if activity dependent processes are involved, bilateral connections would have to shape symmetry, or, temporal constraints could lead to identical patterns on both sides due to their common history of odour exposure.
\end{abstract}

\section{Introduction}

Information processing in the nervous system relies on a very complex interaction between different neuron types. The connections that underlie the computational architecture of the brain are partly genetically programmed, partly established in development via processes such as activity dependent plasticity, and partly remain plastic during the entire life span. Also activity dependent wiring can lead to highly stereotyped functional maps. For example, in the mammalian lateral geniculate nucleus (LGN), afferents from both eyes sort into separate layers. This sorting is accomplished by local synaptic interactions in the LGN, and is driven by spontaneous activity in the ganglion cells of the retinae (Wong et al., 1993; Wong \& Oakley, 1997). Nevertheless, the resultant layering is highly predictable, and bilaterally symmetrical. Further up the visual pathway, in mammals with binocular vision, information from the left and right eye is organized in ocular dominance columns, the layout of which is arranged in alternating stripes. The spacing of these stripes is uniform, while the exact pattern which they form is variable (Anderson et al., 1988). In fact, modelling approaches rely on local, randomly distributed inhomogeneities to trigger the segregation into left-innervated and right-innervated areas for each particular map (Miller et al., 1989; Miller, 1994; Obermayer et al., 1995). In other words, local, activity dependent synaptic interactions lead to functional maps with predetermined overall properties, but local variabilities. Therefore, these maps are not bilaterally symmetrical. Nevertheless, in reversed occlusion experiments in cats an intrinsic cortical component of the columnar layout was found (Gödecke \& Bonhoeffer, 1996).

The mechanisms involved in the interaction between genetically determined and/or activity modulated connections in sensory systems can be well studied in the olfactory system and particularly precisely in that of the bee brain. Olfactory receptor neurons project to the olfactory antennal lobe (AL) in insects or the olfactory bulb in mammals. These structures are organized in glomeruli, which are believed to be the functional units in the first stage of central olfactory processing. The glomerular activity patterns resulting from these projections have been shown to be odour specific (Joerges et al., 1997; Friedrich \& Korsching, 1997). With the exception of locusts (Hansson et al., 1996), single receptor neurons of adult animals always branch in a single glomerulus (Boeckh \& Tolbert, 1993). Also, the projection patterns of receptor neurons all expressing the same putative receptor protein have been shown to converge on a particular glomerulus in mammals (Ressler et al., 1994; Vassar et al., 1994). Consequently, it is the innervation pattern of receptor neurons that creates a spatial map of the odour world accessible to the individual animal (Mombaerts et al., 1996). However, it is still unclear how this map is formed in development. Two extremes are possible: each glomerulus could be predetermined for one (or more) particular (i.e. genetically predetermined) receptor cell family, or an activity dependent sorting mechanism could account for the specific innervation pattern.

An example of a genetically predetermined glomerular map is seen in the macroglomerular complex devoted to sexual pheromone processing in male lepidoptera (Hansson et al., 1992; Hildebrand, 1996). The separation between the main olfactory bulb and the vomeronasal organ in mammals is also genetically determined (Dulac 
\& Axel, 1995). Even within the vomeronasal organ, the apical and the basal regions are innervated by two separate families of receptors (Matsunami \& Buck, 1997; Ryba \& Tirindelli, 1997; Herrada \& Dulac, 1997). For the non-pheromonal system, the evidence is less clear. Odour specific (i.e. odotypic) activity maps look comparably similar in different animals (Johnson \& Leon, 1996; Friedrich \& Korsching, 1997). This suggests, that at least the innervated region, and most likely also the relative position (Mombaerts et al., 1996) of glomeruli are genetically controlled. However, Joerges et al. (1997) found that the interindividual difference is larger for general odours than for odours which are pheromone components. Therefore, activity dependent processes may play a part in shaping the functional map of the antennal lobe. Interestingly, Faber et al. (1997) showed that appetitive learning modifies the measured calcium activity maps. Mercer and co-workers (Winnington et al., 1996; Sigg et al., 1997) have presented morphological evidence for glomerular plasticity in the adult honeybee.

In order to investigate which factors define the functional map of the $\mathrm{AL}$, we asked whether the odotypic pattern is bilaterally symmetrical in the same individual at the level of single glomeruli. We took advantage of several features in the honeybee olfactory system. Olfactory receptors are distributed on the antenna, and project to the AL. Here the number of glomeruli is relatively small $(\approx 160)$ as compared with mammals. Also, their anatomical layout has been shown to be conserved across individuals (Flanagan \& Mercer, 1989), and each glomerulus has been mapped accordingly (Flanagan \& Mercer, 1989; McIlwrath et al., 1997). We have developed a method to record the glomerular activity patterns elicited by stimulation with natural odours (Joerges et al., 1997; Galizia et al., 1997a), and used it to subsequently record the left and the right ALs. We also developed a statistical test for the comparison of bilateral similarity across animals.

We show here, that the activity pattern elicited in the AL of honeybees is bilaterally symmetrical. This holds true for both pheromonal and non-pheromonal substances. Therefore, activity dependent processes local to each $\mathrm{AL}$ are not responsible for the functional identity of the glomeruli. Consequently, the odotypic map must be either genetically predetermined, or dependent on bilaterally coordinated activity processes which lead to an identical map on both sides. Part of this work has been presented in abstract form (Galizia et al., 1997b).

\section{Materials and methods}

\section{Optical recording of the honeybee antennal lobe}

A total of 25 free-flying worker bees (Apis mellifera carnica) were measured in this study. All bees measured in summer came from hives in the field (labelled ' $\mathrm{f}$ ' and ' $\mathrm{fl}$ ' ' in Table 1), bees $8-12$ were all from the same colony (labelled ' $\mathrm{fl}$ ' ' in Table 1). In winter, bees were kept free-flying in an artificial indoor flight chamber at $12 \mathrm{~h}$ light/12 $\mathrm{h}$ dark day cycle (bees labelled with 'c' for 'captive' in Table 1). Animals were experimentally naive, but have learned odours in their colonies and in the field before being captured. Preparation and staining as published elsewhere (Galizia et al., 1997a) has been modified by using not just the isolated head, but the entire bee (Joerges et al., 1997). Briefly, bees were cooled and their heads immobilized. A window was cut into the cuticule to allow access to the brain. Glands and tracheae were removed. The brain was incubated with the calcium indicator calcium-green-2-acetoxymethyl ester (Molecular Probes, Eugene, Oregon) for $1 \mathrm{~h}$, resulting in uniform labelling of the AL. In some preparations, the abdomen was removed to reduce movement artefacts. Images of the AL were taken from the intact brain perfused with bee saline (in $\mathrm{mM}$ ): $\mathrm{NaCl}, 130 ; \mathrm{KCl}, 6$; $\mathrm{MgCl}_{2}, 4 ; \mathrm{CaCl}_{2}$, 5; sucrose, 160; glucose, 25; HEPES, 10; pH 6.7, 500 mOsm (all chemicals from Sigma, Deisenhofen, Germany).

Using a $40 \times$, NA 0.6 LD air objective, one AL was focused, and odour evoked activity patterns were repeatedly measured for up to six different odours in each bee. Then, the contralateral AL of the same animal was centred, taking care to select a comparable view. The external delimitation was used as reference. Again, odour evoked activity patterns were repeatedly measured for different odours.

Under the microscope, the bee was stimulated with a custom built olfactometer. A constant air-stream was switched to an odour-laden one for stimulation, in order to avoid any mechanical stimulus component due to onset or offset of an air stream (Galizia et al., 1997a).

Each measurement consisted of 40 measured frames, at a rate of 2 frames/s. Integration time for each frame was $240 \mathrm{~ms}$. Odourant stimulus was given from the ninth to the 13th frame for a duration of $2 \mathrm{~s}$. Spatial resolution was $51 \times 51$ pixels (binned on chip from the $512 \times 512$ pixels of the cooled CCD camera, Photometrics $\mathrm{CH} 250 \mathrm{~A}$ ), spanning $250 \times 250 \mu \mathrm{m}$ of the AL. Each binned pixel corresponds to $5 \times 5 \mu \mathrm{m}$.

From the raw fluorescence intensity data signals were calculated as $\mathrm{dF} / \mathrm{F}$. The mean fluorescence over the entire 40 frames was taken, for each pixel, as an estimate for F. Dark current of the CCD camera was measured before each experiment (i.e. before each measured bee) and subtracted from the measured fluorescence intensities.

\section{Spatial activity patterns}

Stimulation resulted in local calcium signals. As in this work we were not interested in the temporal progression of the signal, but only in the spatial representation of odours in the AL, a spatial activity pattern was calculated for each stimulus in the following way. For each pixel, the response curve was temporally smoothed, applying a lowpass filter. Then, the difference between frame 13 (peak of the calcium response) and frame 29 ( $8 \mathrm{~s}$ after stimulus onset) was calculated, and taken as a measure of the calcium response at that pixel location for that odour stimulus. No spatial averaging was applied up to this point. These spatial patterns were then spatially low-pass filtered with a 5-pixel $\times 5$-pixel pyramidal filter.

\section{Measuring pattern similarity and bilateral symmetry}

For each animal, the following four similarity indices were calculated: similarity of odour representation for repeated stimulation with the same odour (same odour, ipsilateral), similarity of odour representation for stimulation with the same odour in the contralateral AL (same odour, contralateral), similarity of the representation of different odours in the same AL (different odour, ipsilateral), and similarity of the representation of different odours in the contralateral $\mathrm{AL}$ (different odour, contralateral).

The same-side similarity index was calculated in the following way: for each pair of spatial patterns measured on the same body side (irrespective of left or right), the pixel-wise correlation was calculated. This correlation was calculated on a $40 \times 40$ submatrix, in order to have comparable settings with the different-side measurements (see below). This gives a value between 1 (for perfect match) and -1 (for perfectly complementary activity). All pair-wise correlations for same odour presentation in one animal were averaged to yield the same odour, ipsilateral value, and all pair-wise correlations for different odour presentation were averaged to give the different odour, ipsilateral value.

The different side similarity index was calculated in the following 
TABLE 1. Original data of spatial code similarity for all 25 bees measured. For each bee (rows), and for each of the four comparisons (same odour, ipsilateral and contralateral, and different odours, ipsilateral and contralateral), the mean similarity and its standard deviation are given. $n$ gives the number of comparisons included in the calculation. For example, first line, same odour, ipsilateral: in this bee, seven comparisons of patterns elicited with the same odour are included. In the fourth, seventh, 12 th and 13 th bee no ipsilateral comparisons were possible, because no odour has been repeatedly measured on the same side. The bees are numbered 1-25 in the first column, and labelled ' $\mathrm{f}$ ' for free-flying workers taken from the hive, ' $\mathrm{fl}$ ' for free-flying workers taken all from the same hive, and 'c' for free-flying workers taken from a hive kept in an artificial environment (winter bees)

\begin{tabular}{|c|c|c|c|c|c|c|c|c|c|c|c|c|}
\hline & \multicolumn{3}{|c|}{ Same odour, ipsilat. } & \multicolumn{3}{|c|}{ Same odour, contralat. } & \multicolumn{3}{|c|}{ Different odour, ipsilat. } & \multicolumn{3}{|c|}{ Different odour, contralat } \\
\hline & mean & $\mathrm{SD}$ & $n$ & mean & $\mathrm{SD}$ & $n$ & mean & $\mathrm{SD}$ & $n$ & mean & SD & $n$ \\
\hline 1f & 0.48 & 0.11 & 7 & 0.40 & 0.05 & 12 & 0.11 & 0.14 & 36 & 0.08 & 0.08 & 36 \\
\hline $2 f$ & 0.21 & 0.05 & 3 & -0.24 & 0.06 & 6 & 0.23 & 0.03 & 15 & -0.00 & 0.06 & 12 \\
\hline $3 \mathrm{f}$ & 0.74 & 0.03 & 12 & 0.56 & 0.05 & 24 & 0.42 & 0.07 & 120 & 0.35 & 0.08 & 120 \\
\hline $4 \mathrm{f}$ & & & 0 & 0.64 & 0.01 & 4 & 0.27 & 0.07 & 16 & 0.26 & 0.06 & 16 \\
\hline $5 f$ & 0.71 & 0.01 & 5 & 0.53 & 0.03 & 9 & 0.01 & 0.06 & 41 & 0.01 & 0.07 & 36 \\
\hline $6 f$ & 0.32 & 0.10 & 13 & 0.24 & 0.16 & 19 & 0.11 & 0.08 & 99 & 0.01 & 0.08 & 79 \\
\hline $7 f$ & & & 0 & 0.63 & 0.00 & 2 & 0.54 & 0.03 & 4 & 0.40 & 0.02 & 4 \\
\hline $8 \mathrm{f} 1$ & 0.31 & 0.09 & 6 & 0.49 & 0.11 & 12 & 0.19 & 0.06 & 75 & 0.10 & 0.08 & 60 \\
\hline 9f1 & 0.43 & 0.02 & 11 & 0.40 & 0.12 & 22 & 0.07 & 0.07 & 110 & 0.00 & 0.06 & 110 \\
\hline $10 \mathrm{f} 1$ & 0.12 & 0.06 & 6 & 0.48 & 0.01 & 12 & 0.18 & 0.06 & 75 & 0.27 & 0.08 & 60 \\
\hline $11 \mathrm{f} 1$ & 0.43 & 0.02 & 17 & 0.25 & 0.05 & 20 & 0.16 & 0.07 & 124 & 0.13 & 0.08 & 92 \\
\hline $12 \mathrm{f} 1$ & & & 0 & 0.03 & 0.09 & 6 & 0.19 & 0.04 & 30 & 0.06 & 0.10 & 30 \\
\hline $13 c$ & & & 0 & 0.52 & 0.04 & 4 & 0.14 & 0.06 & 12 & -0.01 & 0.08 & 12 \\
\hline $14 \mathrm{c}$ & 0.74 & 0.05 & 36 & 0.72 & 0.03 & 54 & 0.39 & 0.06 & 270 & 0.32 & 0.08 & 270 \\
\hline $15 \mathrm{c}$ & 0.16 & 0.05 & 7 & 0.28 & 0.07 & 12 & 0.07 & 0.05 & 74 & 0.02 & 0.06 & 60 \\
\hline $16 \mathrm{c}$ & 0.64 & 0.03 & 6 & 0.58 & 0.02 & 12 & 0.39 & 0.07 & 75 & 0.27 & 0.08 & 60 \\
\hline $17 \mathrm{c}$ & 0.68 & 0.01 & 10 & 0.25 & 0.04 & 20 & 0.31 & 0.04 & 80 & 0.21 & 0.06 & 80 \\
\hline $18 \mathrm{c}$ & 0.56 & 0.01 & 6 & 0.18 & 0.02 & 12 & 0.03 & 0.08 & 43 & -0.01 & 0.07 & 44 \\
\hline $19 \mathrm{c}$ & 0.49 & 0.06 & 26 & 0.47 & 0.08 & 32 & 0.35 & 0.11 & 139 & 0.18 & 0.07 & 128 \\
\hline $20 \mathrm{c}$ & 0.41 & 0.05 & 23 & 0.12 & 0.08 & 34 & 0.20 & 0.06 & 134 & 0.12 & 0.06 & 134 \\
\hline $21 \mathrm{c}$ & 0.53 & 0.13 & 82 & 0.47 & 0.07 & 72 & 0.28 & 0.08 & 335 & 0.27 & 0.06 & 252 \\
\hline $22 \mathrm{c}$ & 0.73 & 0.04 & 101 & 0.57 & 0.01 & 94 & 0.18 & 0.04 & 425 & 0.16 & 0.04 & 326 \\
\hline $23 \mathrm{c}$ & 0.58 & 0.05 & 19 & 0.29 & 0.06 & 20 & 0.19 & 0.08 & 101 & 0.13 & 0.07 & 70 \\
\hline $24 \mathrm{c}$ & 0.79 & 0.02 & 89 & 0.30 & 0.05 & 86 & 0.36 & 0.08 & 382 & 0.15 & 0.06 & 346 \\
\hline $25 \mathrm{c}$ & 0.62 & 0.04 & 36 & 0.30 & 0.09 & 48 & 0.12 & 0.07 & 189 & -0.02 & 0.08 & 192 \\
\hline
\end{tabular}

way: activity patterns from the right body side were transformed to their mirror images. In order to allow for a non-perfect match of the field of view in the microscope, a shift in the activity patterns was introduced. Only a $40 \times 40$ pixel submatrix of the measured $51 \times 51$ pixel spatial activity pattern was considered, which leads to a maximal possible shift of $20 \%$. For each pair of ALs (i.e. for each animal), and for each possible relative shift of the submatrices, the mean correlation between all pairs of same odour stimulations was measured. This gave, for each animal, one optimal shift with maximum coefficient of correlation between the left and the right field of view. Taking this relative shift, the similarity measurements were calculated as for the same-side statistics.

Taking the same shift values, but discriminating between the odours used as stimulus, odour-specific similarity indices were calculated in the same way for each individuum.

\section{Statistical testing for bilateral symmetry}

The measured similarity indices were calculated for a total of $n=25$ bees. These are statistically independent measurements, and were statistically tested with ANOVA and Student's $t$-test using the statistical package JMP (SAS institute, Cary, NC, USA).

In order to compare the symmetry of functional maps, odours were changed between experiments. The following odours were tested (in parentheses the number of bees tested with that odour, and the source for the odourant): carnation ( $n=20$, local drug store), citral ( $n=13$, Sigma, Deisenhofen, Germany), geraniol $(n=4$, Sigma), hexanal $(n=10$, Sigma), 2-hexanol $(n=2$, Sigma $), 1$-hexanol $(n=22$,
Sigma), isoamyl acetate ( $n=23$, Sigma), lavender ( $n=2$, local drug store), limonene ( $n=7$, Sigma), linalool ( $n=5$, Sigma), peppermint ( $n=4$, local drug store), octanol $(n=6$, Sigma), orange $(n=3$, local drug store), control air (blank, $n=14$ ).

\section{Results}

\section{Morphological symmetry}

An overview of the opened head capsule of the bee is shown in Fig. 1, seen with the $5 \times$ objective, and a close-up view of the left (Fig. 1B) and right (Fig. 1C) AL. This bee has been stained with the membrane permeant dye RH795 (Molecular Probes). Unlike brains stained with calcium green, which were used for the physiological measurements, ALs stained with RH795 revealed their prominent glomerular pattern. Each glomerulus has an individual shape and size, and the symmetrical arrangement of glomeruli can be seen by comparing the two sides.

\section{Are the measured activity patterns glomerular?}

Spatial activity patterns show a characteristic, odour-specific pattern of activity foci. Figure 2 shows that activated foci correspond to morphological glomeruli. Figure 2A shows a morphological view of a left AL stained with calcium green where the single glomeruli can be seen. Figure $2 \mathrm{~B}$ shows the spatial activity pattern elicited in that $\mathrm{AL}$ when stimulated with isoamyl acetate. The black cross is positioned at the identical location in both images, showing that 

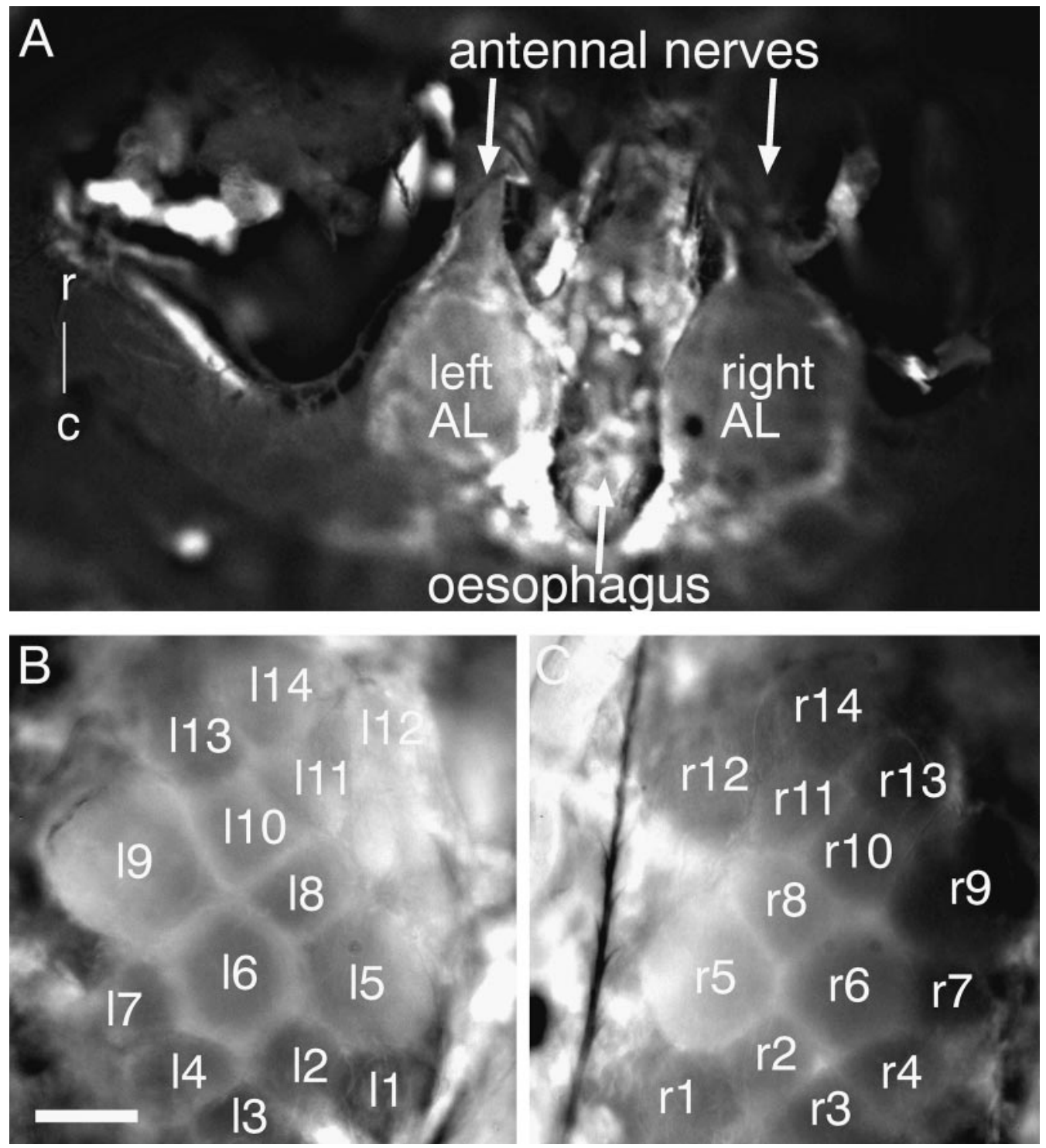

FIG. 1. View of the anatomical symmetry of the glomeruli in the bee AL. The bee brain has been stained with the membrane selective dye RH795, and imaged under the microscope using the same CCD camera as for physiological measurements. (A) View with the $5 \times$ objective. The left and right ALs can be clearly seen, with the antennal nerves pointing up. Between the two ALs the oesophagus is visible. Rostral (r) is up, caudal (c) down. (B,C) View of the left (B) and right $(\mathrm{C}) \mathrm{AL}$ with the $40 \times$ objective, in a view identical to that used in the physiological measurements. Single glomeruli can be recognized by their shape and relative position. To ease comparison, single glomeruli have been given arbitrary numbers, prefixed with 1 (left) or r (right). Scale bar is $50 \mu \mathrm{m}$.

stimulation with isoamyl acetate led to the selective activation of one individual glomerulus in the measured field of view.

\section{Eye inspection reveals that the elicited patterns are symmetrical}

An example of the elicited response patterns to isoamyl acetate, hexanal and 1-hexanol in the two ALs of a single bee is shown in Fig. 4. The figure also shows the response in the same AL to repeated stimulation, and the times when the measurements were taken expressed in hours and minutes after dissection. Each spatial response has been false-colour coded to its own maximum and minimum. Note that the patterns are clearly symmetrical. Single foci can be directly compared with the aid of the superimposed grid.

Small variations, or fluctuations, can also be seen. Particularly prominent, in this bee, is the variability of the response to 1-hexanol. The basic pattern is similar in all four measurements, but a mediocaudal region (bottom right for the right $\mathrm{AL}$ view) is only weakly activated in one left AL measurement, and strongly activated in one right $\mathrm{AL}$ measurement, with intermediate responses in the other two measurements. Therefore, this does not appear to be a left/ right difference, but rather a change of the pattern over time. However, this variability does not appear to be a general property of hexanol (cf. Fig. 6).

\section{A spatial correction is necessary when comparing left and right antennal lobe}

In all experiments reported in this paper, left and right ALs were measured sequentially. The reason is that only the $40 \times$ objective had a numerical aperture sufficient for an acceptable signal to noise ratio, but both ALs could not be imaged simultaneously. The field of view in each AL was selected by eye, taking the external perimeter of the AL as reference. This did not necessarily lead to a perfectly 


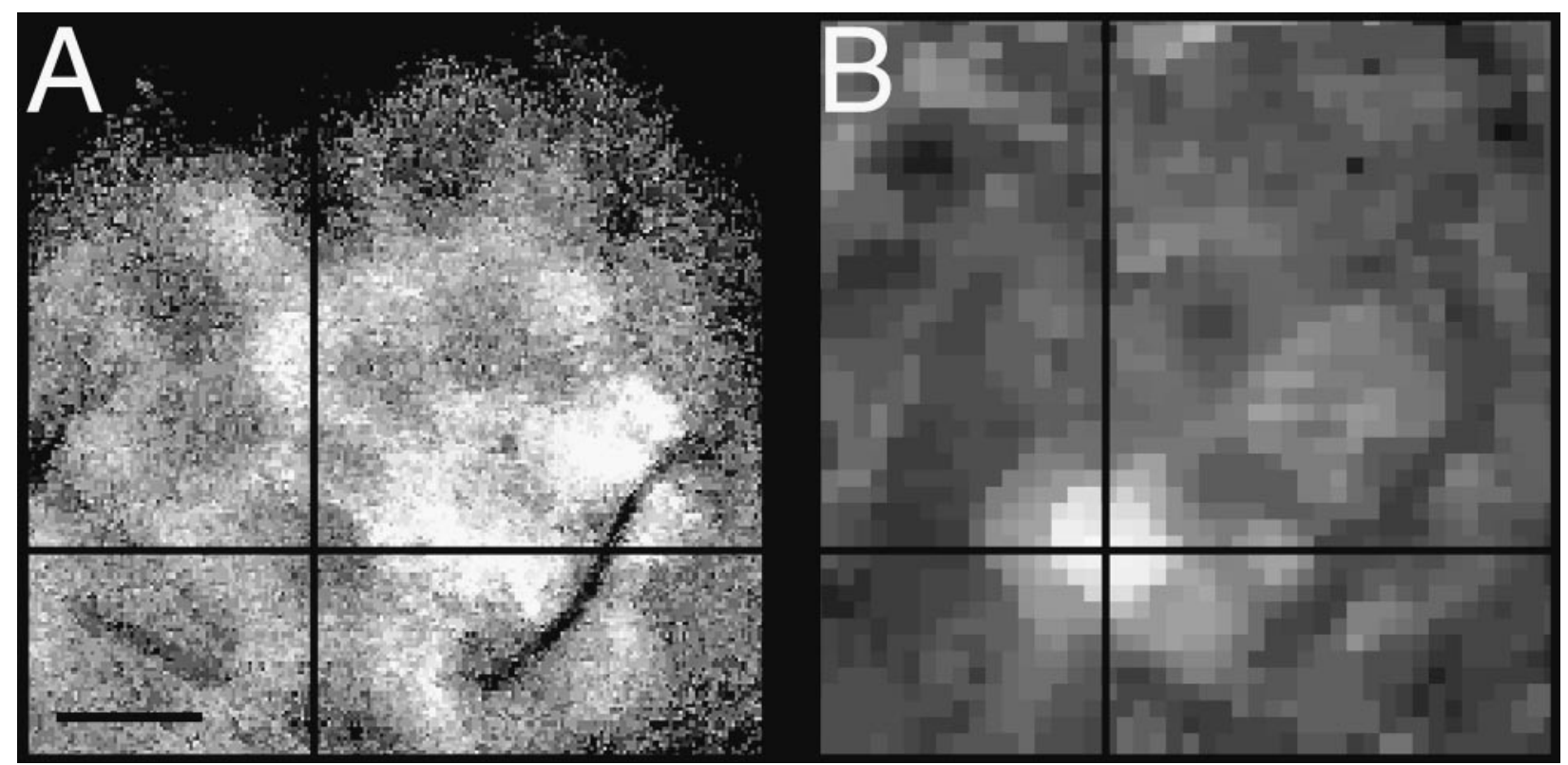

FIG. 2. Activity foci in the physiological measurements correspond to glomeruli. (A) View of an AL stained with calcium green, $40 \times$ objective. Scale bar is $50 \mu \mathrm{m}$. The picture contrast has been digitally enhanced to reveal the glomerular borders, which appear as bright rims. Not all glomerular borders can be seen. (B) False-colour coded activity pattern of the same AL as in A, same field of view. The spatial response pattern to stimulation with isoamyl acetate is represented in the same way as in Fig. 3, using the same grey-scale false-colour sequence. White indicates strong intracellular calcium increase, black no increase. Note that in this experiment, stimulation with isoamyl acetate led to just one activity focus in the field of view. The black cross is positioned at exactly the same location in A and B, revealing that the activity focus in B corresponds exactly to a single glomerulus in A.

corresponding field of view of the two ALs (see Fig. 3, and compare with Fig. 1). In order to correct for small-scale mispositioning, a shift was allowed when quantitatively comparing activity patterns from the right and the left ALs.

An example and its effect upon the measured similarity values is given in Fig. 3. Figure 3A shows the activity map from a left and a right $\mathrm{AL}$ of the same animal, as a response to the odour hexanal. A grid has been superimposed to the pattern. Note that the activity foci are broadly symmetrical, when compared with their relative position in the grid. Figure 3A also shows a mirror image of the left activity map. This allows a one-to-one comparison between the right and the left side. The grid has been chosen to have $80 \%$ sidelength of the measured view, corresponding to $200 \times 200 \mu \mathrm{m}$ in real space. The similarity of the pattern in the grid corresponding to the left AL and the right $\mathrm{AL}$ was measured by calculating the pixel-by-pixel correlation between the two arrays. By shifting the relative positions of the two grids, the correlation changes. Figure $3 \mathrm{~B}$ shows the corresponding correlation values as a function of the relative shift in $x$ and $y$ dimension. With a relative shift of +4 pixels $/+4$ pixels, the highest correlation value $(r=0.86)$ for this $\mathrm{AL}$ is obtained.

\section{Statistical analysis shows that odour coding is bilaterally symmetrical}

Calculating the correlation coefficient between activity patterns gives a useful and solid quantification of their similarity. However, the correlation coefficient does not allow any within-bee statistical testing. The reason is, that the assumed number of degrees of freedom would correspond to the number of pixels minus one (for a $40 \times 40$ array that is 1599), which certainly does not correspond to the true degrees of freedom. The individual pixels of the spatial activity patterns are correlated to each other in a variety of ways, all of which reduce the true number of degrees of freedom, but which are difficult to quantify. The origins of these correlations are manyfold: neighbouring pixels in the CCD camera tend to share some of the noise, i.e. to be correlated for technical reasons. The readout technique leads to a form of correlation which is anisotropic, being greater along the horizontal dimension. Also, the activity patterns are spatially smoothed using a low-pass filter. This adds to lateral correlation. Furthermore, we observe that with the optics used single glomeruli display uniform activity, and do not show any substructures. Therefore, from a biological point of view, the degrees of freedom cannot be more than the numbers of glomeruli imaged, which is in the range of 20-30. All of these arguments would make a direct statistical comparison of spatial activity patterns inherently flawed.

Rather than guessing a fictive number for the degrees of freedom for each image comparison, we chose a different approach. First, for each animal, we found the best necessary shift between the left and right AL, as described in Fig. 3. Then, using this shift, we calculated the mean of all pair-wise correlation coefficients between activity patterns for the following four groups: same odour in the same AL, same odour in the contralateral AL, different odour in the same AL, and different odour in the contralateral AL. We took these four mean correlation values as the similarity indices, i.e. as the measurements for the similarity in the activity patterns in that animal, without assigning them any statistical significance.

We calculated these four similarity indices for a total of 25 individuals. All animals which showed a physiological response on both sides were included, without any preselection of which animals to include. Figure 5 shows the relevant results. Comparison between repeated measurements on one side gives correlation values in the range of about $r=0.50(r=1.0$ would represent a perfect match, with no noise whatsoever). Same stimuli on the contralateral side elicit spatial activity patterns with correlations around $r=0.38$. The results for each bee are given in Table 1.

The statistical analysis leads to the following conclusions: the ANOVA gives a significant difference between the observed groups 


\section{A}

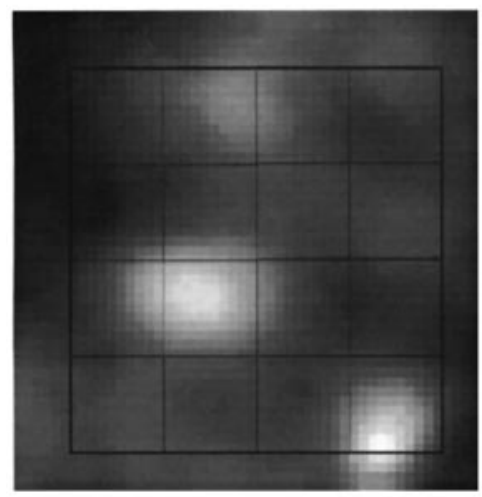

left $A L$

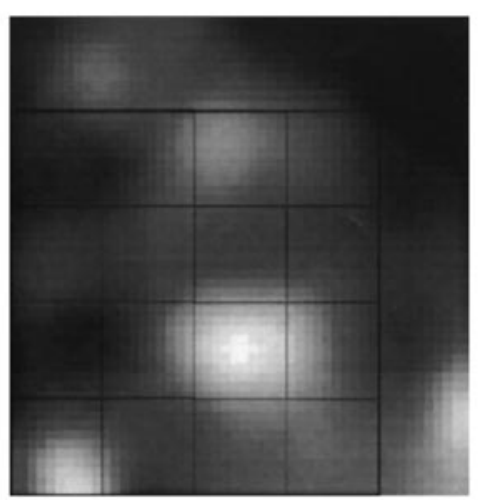

right $\mathrm{AL}$

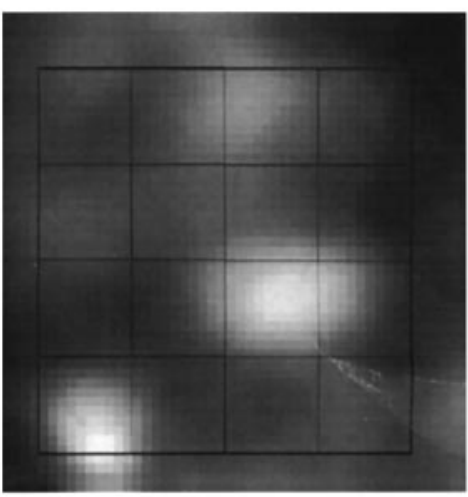

left AL (mirror image)

\section{B}
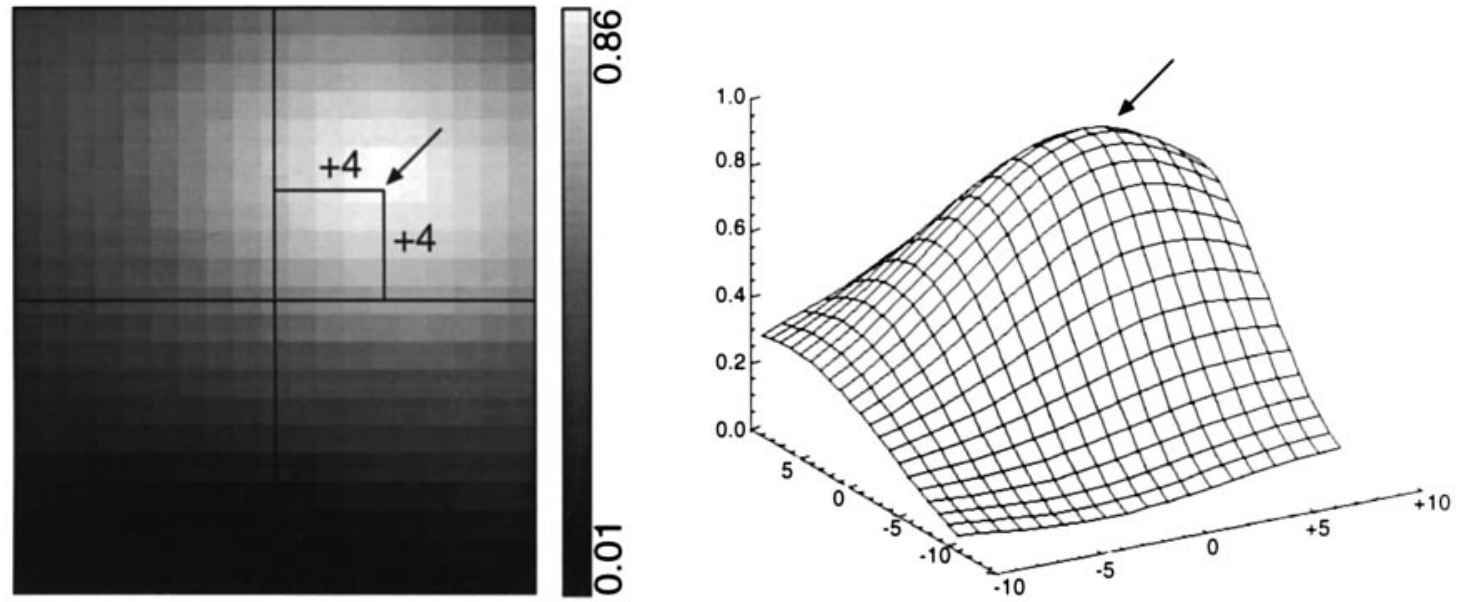

FIG. 3. A shift is necessary when comparing measured right and left AL activity patterns. (A) Activity pattern for the right (middle) and left (left) AL of the same individual, as response to stimulation with hexanal. The right image is the mirror image view of the activity response in the left AL. The increase in intracellular calcium has been coded in grey scales: white denotes highest calcium concentration increase, black no increase. Note that the two images are mirror symmetrical, but slightly shifted in their field of view. As an aid to comparison, a grid (corresponding to $200 \times 200 \mu \mathrm{m}$ in real space) has been superimposed and shifted accordingly. A pixel-to-pixel correlation can be calculated between the image from the right AL and the mirror image view of the left AL. In order to allow for possible shifts, this correlation is calculated only on a subarray, corresponding to the superimposed grid in the figure. (B) Dependence of the calculated correlation upon the relative shift of the subarrays. To the left, a grey-scale coded array shows the calculated correlation (scale to the right, ranging from $r=0.01$ for black to $r=0.86$ for white). The relative shift with the best value ( +4 pixels/ +4 pixels) is marked with an arrow. To the right, the same data is shown as a mesh-plot. It is evident that a single shift value gives the best result. The correlation values in these two plots are the mean correlations for that particular relative shift, calculated for all same odour pairs delivered to that animal on both sides, including the measurement for hexanal shown in Fig. $3 \mathrm{~A}$.

$(P<0.0001, n=25)$. A paired $t$-test between the group 'same odour ipsilateral' and the group 'same odour contralateral' gives no significant difference $(P=0.45)$, while the paired $t$-test between 'different odour contralateral' and 'different odour ipsilateral' is highly significant $(P<0.001)$ (Fig. 5).

Visual inspection of the data in Fig. 5 shows some prominent outliers in the same odour, contralateral group. We inspected some of these measurement sets by hand (bee 2 and bee 12, see Table 1): the worst case (bee 2, with a similarity value between sides of -0.24 ) turned out to be symmetrical, but the fields of view were so badly chosen that a shift of over $30 \%$ would have been necessary in the horizontal direction in order match the two sides. As the maximum shift allowed in the automated matching algorithm is $20 \%$, the observed 'similarity index' lies almost in the anticorrelation range.
The head capsule in bee 12 was slightly rotated. Despite knowing the reasons for their bad performance, we did not exclude these cases from the statistical analysis reported in the figures. Excluding these two data points confirms the statistical difference between 'different odour, contralateral' and 'different odour, ipsilateral' $(P<0.001)$ and between 'different odour, ipsilateral' and 'same odour, contralateral' $(P<0.001)$. The lack of statistical difference between 'same odour, contralateral' and 'same odour, ipsilateral' (i.e. the bilateral symmetry of the evoked activity patterns) is increased $(P=0.60$, as opposed to $P=0.45$ reported above when these outliers are included).

\section{Bilateral symmetry is equal for all tested odours}

We analysed the bilateral symmetry separately for all odours tested (Fig. 6). We deliberately included odours that are environmental 
flavours (like hexanol, hexanal and peppermint), and odours that have a role as non-sexual pheromones in honeybees workers (e.g. citral, isoamyl acetate and geraniol). In the group of non-pheromones, both pure chemicals (e.g. 1-hexanol, 2-hexanol) and complex blends (e.g. orange, carnation) have been included. In bees, unlike in

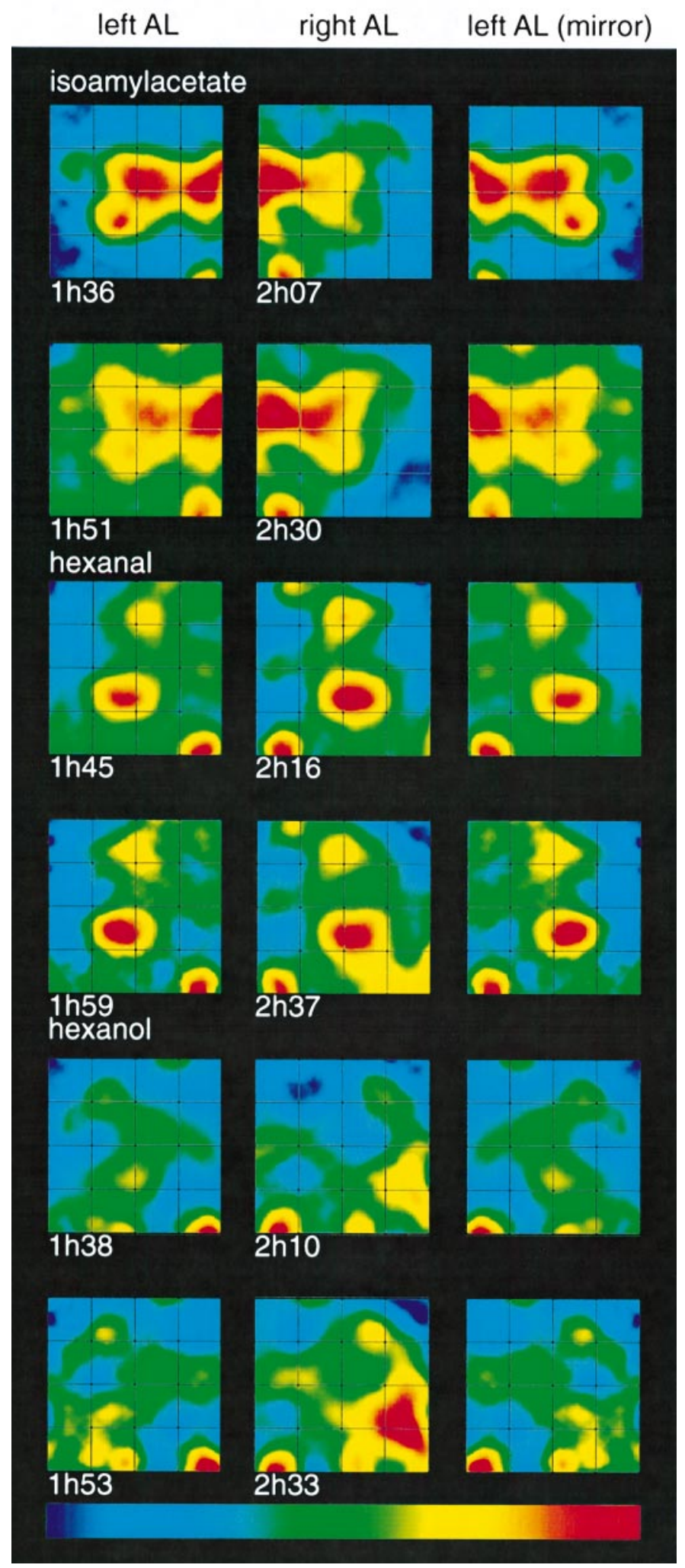

mammals (Bargmann, 1997), the representation of non-sexual pheromone components is overlapping with the representation of nonpheromonal odours: by comparing the responses with isoamyl acetate, a sting pheromone component, in Fig. 4 with the responses to hexanal or hexanol, which have no pheromonal capacity, in the same figure, it can be stated that they share several glomeruli in their code. No statistical difference could be detected between any of the odours (ANOVA, $P=0.51$ ). Therefore, bilateral symmetry is independent of which odour is coded.

The similarity index for stimulation with air (blank control) is also included in Fig. 6. The mean similarity index is 0.07 , with a standard error of 0.07 , which is expected for random data. Including this group into the statistical analysis for odour difference, the ANOVA gives a value of $P=0.02$, indicating that there is a significant difference in the groups observed. In other words, the similarity index between controls (i.e. no similarity between the response to air on the left and the right $\mathrm{AL}$, mean similarity index 0.07) is statistically different from the similarity index between the left and right odour evoked activity patterns.

\section{Discussion}

In this paper, we statistically compare the glomerular activity code for odours in the olfactory ALs of honeybees. We show that this code is bilaterally symmetrical in the ALs of the same animals, and that this symmetry is independent of the odour tested, i.e. that all odours are symmetrically coded.

\section{Methodological aspects: signal source}

Spatial activity patterns were measured using intracellular calcium concentration as an indicator for neural activity (Joerges et al., 1997; Galizia et al., 1997a). The observed increases in intracellular calcium correspond to neurally activated glomeruli. This can be shown by the match of the activity foci with the perimeter of single glomeruli, as sometimes seen with calcium green staining (Fig. 2), or after subsequent staining with RH795 (Joerges et al., 1997). However, we do not know the exact contribution of the various cellular elements in the AL to the measured pattern. The AM ester, which allows the dye

FIG. 4. Odour elicited activity patterns are broadly symmetrical, but show some variability both after repeated measurement, and between body sides. The figure shows the elicited intracellular calcium increase upon repeated stimulation with different odourants in the right and the left AL of a single worker bee. Taking the columns from left to right, the response elicited in the left $\mathrm{AL}$ is shown, than the response in the right $\mathrm{AL}$, and then again the response in the left AL, but displayed as mirror image. Therefore, the two external columns show the identical data set, but allow to compare the central column (right AL) as a mirror image with the left column (left AL), or as a same-field view with the right column (left AL, mirror image). All images are single measurements of elicited responses. Below each frame in the central and left column, the time after preparation is given in hours and minutes. The first measurement taken is the upper response to isoamyl acetate in the left $\mathrm{AL}, 1 \mathrm{~h}$ and $36 \mathrm{~min}$ after preparation. The last is $\approx 1 \mathrm{~h}$ later, $2 \mathrm{~h}$ and $37 \mathrm{~min}$ after preparation, the second response to 1-hexanol in the right AL. For each of the odours isoamyl acetate, hexanal and hexanol two separate measurements for the right and for the left AL are shown, allowing to compare the consistency of the response in one side with the symmetry between sides. A grid has been superimposed in order to ease the comparison of the spatial activity patterns. Note the high degree of similarity both between the two sides and comparing repeated stimulation. All images are false-colour coded (see colour bar at the bottom) to the entire range, i.e. the maximum activity in each measurement is coded as red, the minimum is coded as blue. Therefore, spatial patterns can easily be compared, absolute response values however, cannot. The two sides have been relatively shifted, as shown in Fig. 3, in order to get the best match. 
to permeate cell membranes, could stain all cells in the AL: axons of receptor neurons (there are about 60000 to each AL, Esslen \& Kaissling, 1976), multiglomerular interneurons (about 4000 in each AL, Witthöft, 1967), multi- and uniglomerular projection neurons (about 500, Bicker et al., 1993), and glial cells. Afferent receptor cells could contribute to the calcium signal with their presynaptic calcium influx necessary for transmitter release. Antennoglomerular

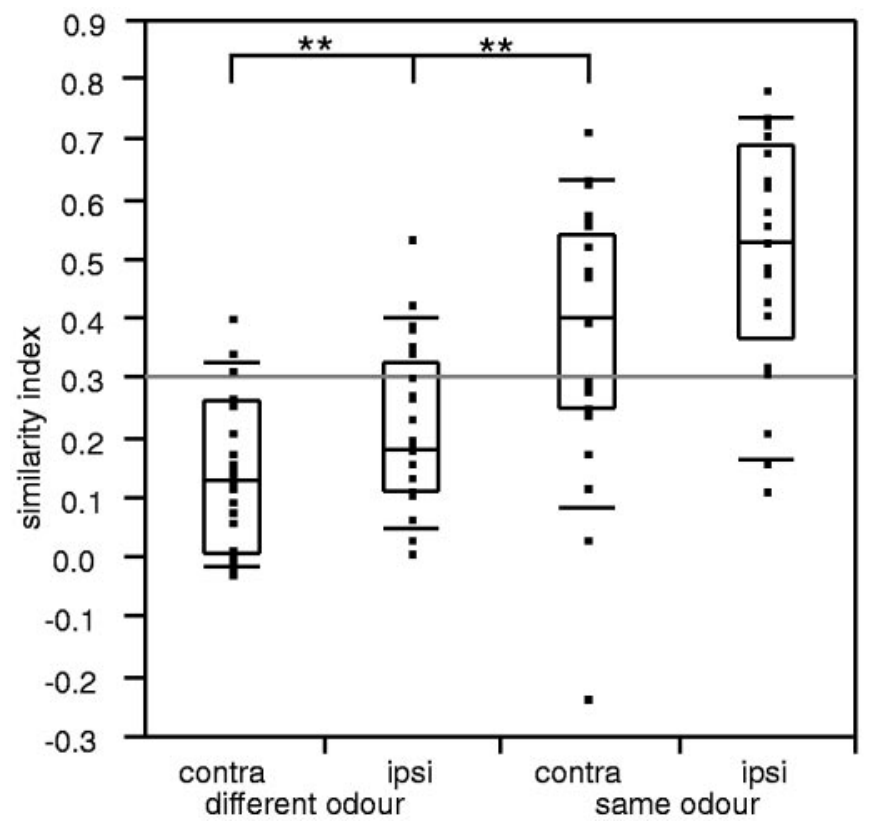

FIG. 5. Statistical comparison of the similarity of spatial activity patterns within and between body sides. For each category, all measurement points are plotted, superimposed with a box plot. The box plot gives the median, $25 \%$ quantile and $75 \%$ quantile in the box, and the $10 \%$ quantile and $90 \%$ quantile as dashes. The horizontal line represents the overall mean for all points. The ANOVA gives a significant difference between the observed groups $(P<0.0001)$. Using pair-wise comparisons, there is no significant difference between same odour, ipsilateral and same odour, contralateral. $* * P<0.001$, paired $t$-test. projection cells could contribute to the calcium signal via dendritic calcium influx. Interneurons have both compartments (presynaptic terminals and dendritic integration segments) within the AL. We assume that our signal is a composite signal of different cellular compartments. One portion of the measured patterns probably reflects activity of the presynaptic terminals of receptor cells, for the following reasons: We only measure about 30 of the $\approx 160$ glomeruli of the honeybee. All of the imaged glomeruli belong to the T1 tract of the antennal nerve, as can be shown by comparison with an atlas of the AL glomeruli (Flanagan \& Mercer, 1989; McIlwrath et al., 1997). In all glomeruli of the T1 tract, the innervating axons strongly branch in the rind of each glomerulus (Arnold et al., 1985). Therefore, a change in fluorescence in the core may be masked by the fluorescence in the rind, whereas the fluorescence in the rind can be directly measured with the CCD camera. Calcium changes in the glomerular rind are likely to be due mainly to presynaptic activity in the receptor neurons, and postsynaptic activity in the interneurons. If a component of the signal was due to intracellular calcium changes in glial cells, these would still reflect local neural activity. The fact that odourant mixtures lead to glomerular interactions reveals that an important component is also due to intraglomerular communication, i.e. to interneurons (Joerges et al., 1997). Therefore, the measured activity patterns reflect the functional innervation pattern to the olfactory glomeruli in the $\mathrm{AL}$, as modified by lateral connections of the interneurons.

\section{Methodological aspects: evaluation of symmetry, statistics}

When investigating functional bilateral symmetry, the first prerequisite is for the underlying structures to be anatomically symmetrical. The AL of the honeybee is composed of about 160 glomeruli, which have been individually identified and mapped (Arnold et al., 1985; Flanagan \& Mercer, 1989; McIlwrath et al., 1997). Therefore, a morphological comparison between the left and right $\mathrm{AL}$ appears feasible.

Indeed, Fig. 1 shows such an anatomical comparison using the same set-up as the one used in the physiological measurements. The figure shows that there is a very good match of the two sides, but it also shows that the match is not perfect. Partly, this is due to unequal choice of the field of view. For example, in Fig. 1C glomerulus r9

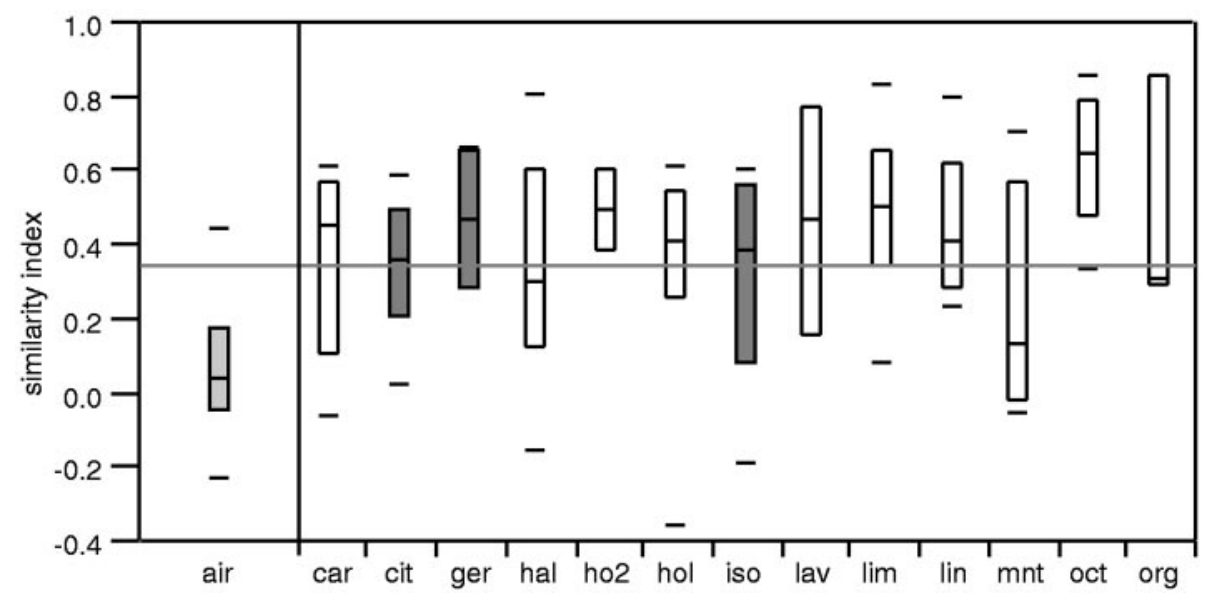

FIG. 6. Statistical comparison of the symmetry for different odours. For each odour, the box plots are given representing the bilateral symmetry values for that odour (median, 10\%, 25\%, 75\% and 90\% quantiles, the extreme quantiles only where there are sufficient observations). The line represents the overall mean value. The ANOva gives no significant differences between the similarity values for the different odours $(P=0.51)$. To the left, bilateral symmetry values for stimulation with air is also given. These values range around the mean of 0.07 , which reflects the randomness of the responses to air control (i.e. no response). Boxes for citral, geraniol and isoamyl acetate are shaded to highlight the role of these odours as pheromones: citral and geraniol are components of the Nasanov gland pheromone, isoamyl acetate is a component of the alarm pheromone associated with one of the sting glands (Free, 1987). 
reaches the right side of the image, whereas 19 in Fig. 1B does not. In our experiments, we could only use the external perimeter of the $\mathrm{AL}$ as a reference for positioning the microscope, which often led to slight lateral shifts (compare with Fig. 3). We therefore corrected for lateral shifts in the statistical analysis. We sometimes also observed that the two ALs were slightly rotated, probably due to a slight misalignment of the body axis to the microscope axis, which leads to a rotation of the two views (data not shown). No corrections for rotational differences were introduced in the statistical analysis. As reported in the results section, for some ALs a shift greater than $20 \%$ would have been necessary to match the two sides. We decided not to enlarge the allowed range for two reasons: $20 \%$ corresponds to $50 \mu \mathrm{m}$, which is about the size of one glomerulus. First, allowing for shifts greater than one glomerulus would open the possibility of outof-register matches between the two ALs; second, increasing the shift also means reducing the overlapping area, i.e. the number of glomeruli compared. It is interesting to see, however, that the case where $30 \%$ shift would have been necessary gives the worst similarity index of all comparisons, which indicates that mismatching activity patterns leads to anticorrelations.

\section{Comparative aspects: reports of symmetry in other species}

Several approaches have been applied to unravel the spatial olfactory code in various animals. Some are of anatomical nature, investigating the innervation pattern of sensory axons, other functional, using radioactive 2-deoxy-glucose (2DG) marking of activated regions, or activity measurements with calcium or voltage sensitive dyes.

In rodents, the projection patterns of genetically defined afferents to the olfactory bulb have been shown to be bilaterally symmetrical and conserved between animals in their relative positions (Vassar et al., 1994; Ressler et al., 1994; Mombaerts et al., 1996). However, unlike the bee, no complete mapping of the glomeruli is possible. A conserved pattern of the anatomical projection has been shown in zebrafish (Baier \& Korsching, 1994).

Using 2DG, Johnson \& Leon (1996) report the physiological response to be bilaterally symmetrical in rats. They also report that these patterns are different between animals, unless they come from the same litter (Johnson \& Leon, 1996). Friedrich \& Korsching (1997) report that the activity pattern elicited by olfactory stimulation in the olfactory bulb of zebrafish is comparable from one animal to the next. This would also argue for a bilaterally symmetrical pattern. In Drosophila melanogaster, unlike the honeybee, most receptor axons branch in both ALs (Stocker, 1994). Functional bilateral symmetry has been reported in the AL of Drosophila (Rodriguez, 1988) using 2DG staining, suggesting that bilaterally staining neurons branch in homologous glomeruli. However, to our knowledge, no statistical evaluation has been performed up to now for the comparison of spatial olfactory activity maps.

\section{Developmental aspects: how does symmetry come about?}

We show here that the functional glomerular pattern is bilaterally symmetrical for pheromones and non-pheromones alike. The measured activity patterns reflect the functional innervation pattern of the glomeruli in the $\mathrm{AL}$ by receptor neurons, as modified by lateral connections of the interneurons.

The molecular mechanisms for target recognition of olfactory receptor cells, and for developmental wiring of local interneurons, are not well understood. In principle, two mechanisms may be involved: genetic determination or activity dependent processes.

Axons may innervate the $\mathrm{AL}$ and find their target using activity dependent mechanisms which rely on local activity patterns, in a way reminiscent of the segregation of ocular dominance columns in the visual cortex. Genetic instructions could determine the course glomerular pattern (for example, which axons innervate each of the four branches, $\mathrm{T} 1-\mathrm{T} 4$, of the antennal nerve), while the fine grain pattern would be activity dependent. In such a case, the selection of a particular glomerulus by a receptor cell family would be the outcome of a competitive interaction as a consequence of external stimulation where local perturbations or irregularities would determine which glomerulus becomes innervated by which receptor cell family. Therefore, we would expect the bilateral pattern not to be functionally symmetrical, and the results from our experiments exclude this possibility.

On the other extreme, the innervation pattern could be totally genetically determined. In this case, the functional maps would be bilaterally symmetrical. We would also postulate that the map be identical for different individuals, possibly for all individuals of Apis mellifera, or at least for all bee sisters with an identical genetical background. The functional glomerular map for pheromones has been shown to be conserved between individuals (Joerges et al., 1997), and is bilaterally symmetrical (Fig. 6); therefore, this map may be under genetical control.

However, activity maps differ between individuals for non-pheromones (Joerges et al., 1997), despite being bilaterally symmetrical within one animal (Fig. 6). Consequently, both a genetic and an activity dependent component are involved in functional glomerular mapping. In order to explain how an activity dependent component could create bilateral symmetry, some sort of temporal constraints or bilateral comparisons have to be postulated. Temporal constraints could mean that the developmental sequence of odours being experienced by the bee influences the functional map of the AL. Such a scenario would also imply that the olfactory code for pheromones will be more similar between animals than the code for environmental odours. Indeed, the comparison of the activity patterns elicited by citral (a pheromone) and by hexanol (a flower odour component) in different individuals reveals the pattern for citral to be conserved, and the pattern for hexanol to be more variable (Joerges et al., 1997). However, in our study, the symmetry of olfactory maps appeared not to depend on the biological meaning of an odour (Fig. 6). Pheromones are produced by the bees in the hive and therefore will always belong to the first odours encountered in development, which could lead to their representation being more similar between individuals than the representation of non-pheromonal odours.

How might the odotypic excitation patterns be synchronized in the two ALs? In the honeybee, there is only a very small direct projection between the two ALs (Arnold et al., 1985; Mobbs, 1985), which is GABAergic (Schäfer \& Bicker, 1986). There are, however, interneurons which connect homologous glomeruli in the two ALs; these are generally neurons from the suboesophageal ganglion (R. Abel, unpublished observations). Furthermore, several unpaired neurons innervate both ALs. One of these neurons was found to implement the reinforcing function during olfactory learning (Hammer, 1993). The synchronous activation of both ALs (and other paired structures in the bee brain) could serve the developmental function to equalize synaptic strengths in corresponding anatomical structures. Therefore, the role of these neurons could be to guarantee for developmental symmetry. As we have never observed an excitation in an AL when the antenna was wet or damaged in the experiment, even if the contralateral AL gave good signals, these interneurons are unlikely to contribute to the measured calcium signal. They could, however, modulate the glomerular activity pattern. 


\section{Functional significance of bilateral symmetry}

The activity pattern which we measure as a response to an odour stimulus in the glomeruli of the AL represents the spatial component of the functional code for that particular odour. This code could be the information that the brain uses in identifying an odour, be it a pheromone which often leads to a stereotyped behaviour, or an environmental odour which may be associated with a particular meaning, for example after appetitive learning during foraging. Several brain structures are involved in the choice of behavioural responses and in learning, including the ALs themselves, and in particular the mushroom bodies (Menzel \& Müller, 1996), where most axons leaving the ALs project to. Classical conditioning of an odour with a sucrose reward with one antenna covered leads to a memory accessible only to the trained side, i.e. exposing the untrained antenna to the learned odour does not elicit a conditioned response (Masuhr \& Menzel, 1972). Complex forms of learning, like olfactory blocking, rely on sensory input from both antennae (Thorn \& Smith, 1997). A symmetrical code in the ALs may be an important aspect for reliable odour recognition at the level of the mushroom bodies.

Also, as has been shown in the ant Lasius fuliginosus when following a trail, the brain relies on the comparison between the sensory input from the left and right antenna (Hangartner, 1967). Osmotropotactic behaviour has also been reported in honeybees (Martin, 1964). For osmotropotactic behaviour, a symmetrical map in the ALs may be useful to compare concentration gradients or temporal delays between the body sides.

\section{Future prospects}

We have shown the olfactory code to be bilaterally symmetrical in Apis mellifera. An important aspect left to investigate is the role of early experience in shaping the code. In order to compare the code between individuals efficiently, a functional mapping on to anatomically identified glomeruli would be necessary. As it is possible to classify anatomically the glomeruli, such an approach appears to be feasible. That would also open the way to experiments where the early experience of the bee is manipulated, either by swamping the hive with a particular odour, thus leading to a permanent saturation of all receptors for that odour, or by manipulations such as early odour deprivation. These experiments will tell us more about the developmental mechanisms underlying target finding of sensory axonal projections in the bee AL.

\section{Acknowledgements}

Thanks to Regina Abel, Till Faber, Jasdan Joerges and Ulrike Schröter for help with the manuscript. Thanks to Dedo Koppitz for excellent mechanical expertise. Supported by DFG Me 365/21-1.

\section{Abbreviations}

\section{AL}

\section{antennal lobe}

LGN lateral geniculate nucleus

2DG 2-deoxyglucose

\section{References}

Anderson, P.A., Olavarria, J. \& Van Sluyters, R.C. (1988) The overall pattern of ocular dominance bands in cat visual cortex. J. Neurosci., 8, 2183-2200. Arnold, G., Masson, C. \& Budharugsa, S. (1985) Comparative study of the antennal lobes and their afferent pathway in the worker bee and the drone (Apis mellifera). Cell Tissue Res., 242, 593-605.

Baier, H. \& Korsching, S. (1994) Olfactory glomeruli in the zebrafish form an invariant pattern and are identifiable across animals. J. Neurosci., 14, 219-230.

Bargmann, C.I. (1997) Olfactory receptors, vomeronasal receptors, and the organization of olfactory information. Cell, 90, 585-587.
Bicker, G., Kreissl, S. \& Hofbauer, A. (1993) Monoclonal antibody labels olfactory and visual pathways in Drosophila and Apis brains. J. Comp. Neurol., 335, 413-424.

Boeckh, J. \& Tolbert, L.P. (1993) Synaptic organization and development of the antennal lobe in insects. Microsc. Res. Tech., 24, 260-280.

Dulac, C. \& Axel, R. (1995) A novel family of genes encoding putative pheromone receptors in mammals. Cell, 83, 195-206.

Esslen, J. \& Kaissling, K.-E. (1976) Zahl und Verteilung antennaler Sensillen bei der Honigbiene (Apis mellifera L.). Zoomorphologie, 83, 227-251.

Faber, T., Joerges, J., Galizia, G., Küttner, A. \& Menzel, R. (1997) Learning induced changes of activity in the bee's brain monitored by optical imaging: first results. In Elsner, N. \& Wässle, H. (eds) From Membrane to Mind. Göttingen Neurobiology Report 1997, p. 156. G. Thieme, Stuttgart.

Flanagan, D. \& Mercer, A.R. (1989) An atlas and 3-D reconstruction of the antennal lobes in the worker honey bee, Apis mellifera L. (Hymenoptera: Apidae). Int. J. Insect Morphol. Embryol., 18, 145-159.

Free, J.B. (1987) Pheromones of Social Bees. Chapman \& Hall, London.

Friedrich, R.W. \& Korsching, S.I. (1997) Combinatorial and chemotopic odorant coding in the zebrafish olfactory bulb visualized by optical imaging. Neuron, 18, 737-752.

Galizia, C.G., Joerges, J., Küttner, A., Faber, T. \& Menzel, R. (1997a) A semi-in-vivo preparation for optical recording of the insect brain. J. Neurosci. Methods, 76, 61-69.

Galizia, C.G., Nägler, K. \& Menzel, R. (1997b) Spatial olfactory mapping is bilaterally symmetric in insect antennal lobes. Soc. Neurosci. Abstr., 23, 1826 (Abstr.).

Goedecke, I. \& Bonhoeffer, T. (1996) Development of identical orientation maps for two eyes without common visual experience. Nature, 379, 251-254.

Hammer, M. (1993) An identified neuron mediates the unconditioned stimulus in associative olfactory learning in honeybees. Nature, 366, 59-63.

Hangartner, W. (1967) Spezifität und Inaktivierung des Spurpheromons von Lasius fuliginosus Latr. und Orientierung der Arbeiterinnen im Duftfeld. Z. Vergl. Physiol., 57, 103-136.

Hansson, B.S., Ljungberg, H., Hallberg, E. \& Löfstedt, C. (1992) Functional specialization of olfactory glomeruli in a moth. Science, 256, 1313-1315.

Hansson, B.S., Ochieng, S.A., Grosmaitre, X., Anton, S. \& Njagi, P.G.N. (1996) Physiological responses and central nervous projections of antennal olfactory receptor neurons in the adult desert locust, Schistocerca gregaria (Orthoptera: Acrididae). J. Comp. Physiol. A, 179, 157-167.

Herrada, G. \& Dulac, C. (1997) A novel family of putative pheromone receptors in mammals with a topographically organized and sexually dimorphic distribution. Cell, 90, 763-773.

Hildebrand, J.G. (1996) Olfactory control of behavior in moths: Central processing of odor information and the functional significance of olfactory glomeruli. J. Comp. Physiol. A, 178, 5-19.

Joerges, J., Kuettner, A., Galizia, C.G. \& Menzel, R. (1997) Representations of odours and odour mixtures visualized in the honeybee brain. Nature, 387, 285-288.

Johnson, B.A. \& Leon, M. (1996) Spatial distribution of $\left[{ }^{14} \mathrm{C}\right] 2$-deoxyglucose uptake in the glomerular layer of the rat olfactory bulb following early odor preference learning. J. Comp. Neurol., 376, 557-566.

Martin, H. (1964) Zur Nahorientierung der Biene im Duftfeld. Zugleich ein Nachweis für die Osmotropotaxis bei Insecten. Z. vergl. Physiol., 48, 481-533.

Masuhr, T. \& Menzel, R. (1972) Learning experiments on the use of sidespecific information in the olfactory and visual system in the honeybee of Apis mellifica. In Wehner, R. (ed.), Information Processing in the Visual Systems of Arthropods, pp. 315-322. Springer-Verlag, Berlin.

Matsunami, H. \& Buck, L.B. (1997) A multigene family encoding a diverse array of putative pheromone receptors in mammals. Cell, 90, 775-784.

McIlwrath, S.L., Galizia, C.G. \& Menzel, R. (1997) A 3-dimensional volumetric atlas of the antennal lobe of the honeybee, Apis mellifera. In Elsner, N. \& Wässle, H. (eds), From Membrane to Mind. Göttingen Neurobiology Report 1997, p. 693. G. Thieme, Stuttgart.

Menzel, R. \& Müller, U. (1996) Learning and memory in honeybees: from behavior to neural substrates. Аппи. Rev. Neurosci., 19, 379-404.

Miller, K.D. (1994) A model for the development of simple cell receptive fields and the ordered arrangement of orientation columns through activitydependent competition between ON- and OFF-center inputs. J. Neurosci., 14, 409-441.

Miller, K.D., Keller, J.B. \& Stryker, M.P. (1989) Ocular dominance column development: Analysis and simulation. Science, 245, 605-615. 
Mobbs, P.G. (1985) Brain structure. In Kerkut, G.A. \& Gilbert, L.I. (eds), Comprehensive Insect Physiology Biochemistry and Pharmacology. 5: Nervous System: Structure and Motor Function, pp. 299-370. Pergamon Press, Oxford.

Mombaerts, P., Wang, F., Dulac, C., Chao, S.K., Nemes, A., Mendelsohn, M., Edmondson, J. \& Axel, R. (1996) Visualizing an olfactory sensory map. Cell, 87, 675-686.

Obermayer, K., Sejnowski, T. \& Blasdel, G.G. (1995) Neural pattern formation via a competitive Hebbian mechanism. Behav. Brain Res., 66, 161-167.

Ressler, K.J., Sullivan, S.L. \& Buck, L.B. (1994) Information coding in the olfactory system: Evidence for a stereotyped and highly organized epitope map in the olfactory bulb. Cell, 79, 1245-1255.

Rodriguez, V. (1988) Spatial coding of olfactory information in the antennal lobe of Drosophila melanogaster. Brain Res., 453, 299-307.

Ryba, N.J.P. \& Tirindelli, R. (1997) A new multigene family of putative pheromone receptors. Neuron, 19, 371-379.

Schäfer, S. \& Bicker, G. (1986) Distribution of GABA-like immunoreactivity in the brain of the honeybee. J.Comp. Neurol., 246, 287-300.

Sigg, D., Thompson, C.M. \& Mercer, A.R. (1997) Activity-dependent changes to the brain and behavior to the honey bee, Apis mellifera (L.). J. Neurosci., 17, 7148-7156.

Stocker, R.F. (1994) The organization of the chemosensory system in Drosophila melanogaster: a review. Cell Tissue Res., 275, 3-26.

Thorn, R.S. \& Smith, B.H. (1997) The olfactory memory of the honeybee Apis mellifera. 3. Bilateral sensory input is necessary for induction and expression of olfactory blocking. J. Exp. Biol., 200, 2045-2055.

Vassar, R., Chao, S.K., Sitcheran, R., Nunez, J.M., Vosshall, L.B. \& Axel, R. (1994) Topographic organization of sensory projections to the olfactory bulb. Cell, 79, 981-991.

Winnington, A.P., Napper, R.M. \& Mercer, A.R. (1996) Structural plasticity of identified glomeruli in the antennal lobes of the adult worker honey bee. J. Comp. Neurol., 365, 479-490.

Witthöft, W. (1967) Absolute Anzahl und Verteilung der Zellen im Hirn der Honigbiene. Z. Morph. Tiere, 61, 160-184.

Wong, R.O.L. \& Oakley, D.M. (1997) Changing patterns of spontaneous bursting activity of on and off retinal ganglion cells during development. Neuron, 16, 1087-1095.

Wong, R.O.L., Meister, M. \& Shatz, C.J. (1993) Transient period of correlated bursting activity during development of the mammalian retina. Neuron, 11, 923-938. 